\title{
NOVEDADES TAXONÓMICAS EN GALIANTHE Y BORRERIA (RUBIACEAE-SPERMACOCEAE)
}

\author{
por ELSA L.CABRAL ${ }^{1}$ Y NÉLIDA M. BACIGALUPO²
}

\section{Summary}

\begin{abstract}
Three new species, Galianthe andersonii E.L.Cabral, Galianthe macedoi E.L.Cabral, Galianthe reitzii E.L.Cabral are described and illustrated and two new combinations, Galianthe peruviana (Pers.) E.L.Cabral and Borreria crispata (K. Schum.) E.L.Cabral \& Bacigalupo, are presented. A new status and a new name are proposed for Borreria burchelli;; taxonomic observations are given about Borreria tenera DC.
\end{abstract}

Key words: taxonomy, Galianthe, Borreria, Rubiaceae, Spermacoceae.

Palabras claves: taxonomía, Galianthe, Borreria, Rubiaceae, Spermacoceae.

Durante la revisión de la tribu Spermacoceae se han encontrado algunas novedades para los géneros Galianthe y Borreria en Sudamérica. En Galianthe subgen. Galianthe (Cabral \& Bacigalupo, 1997) se describen tres especies nuevas provenientes de Brasil: G. andersonii E.L.Cabral sp. nov. (Minas Gerais), G. macedoi E.L.Cabral sp. nov. (Goiás) y G. reitzii E.L.Cabral sp. nov. (Santa Catarina) y una nueva combinación Galianthe peruviana (Pers.) E.L.Cabral comb. nov. En Borreria se realizan cambios taxonómicos en las siguientes especies: B. crispata (K.Schum.) E.L.Cabral \& Bacigalupo, stat. et comb. nov., B. burchellii E.L.Cabral \& Bacigalupo, stat. et nom. nov., y se dan a conocer nuevos sinónimos de B. tenera DC.

Galianthe Griseb. es un género americano perteneciente a la tribu Spermacoceae, recientemente rehabilitado por Cabral (1991). Se caracteriza principalmente por sus inflorescencias

\footnotetext{
${ }^{1}$ Facultad de Ciencias Exactas, Naturales y Agrimensura, UNNE. Instituto de Botánica del Nordeste, Casilla de Correo 209, 3400, Corrientes, Argentina. Este trabajo es parte de la tesis doctoral a ser presentada en la Universidad Nacional del Nordeste

${ }^{2}$ Instituto de Botánica Darwinion, Casilla de Correo 200, 1642 San Isidro, Argentina, co-directora de tesis.
}

amplias, tirsoideas y sus flores heterostilas. Está representado aproximadamente por 50 especies agrupadas en dos subgéneros Galianthe y Ebelia (Cabral \& Bacigalupo, 1997). De éstas, unas 40 exclusivamente sudamericanas, pertenecen al subgénero Galianthe que reúne las especies con frutos de mericarpos dehiscentes. A este subgénero pertenecen los taxones que se describen a continuación.

1. Galianthe andersonii E.L.Cabral sp. nov. Fig. 1

Suffrutex ramosissimus, $60-100 \mathrm{~cm}$ alt. Caules quadrangulares rubescens, puberulis vel glabrescentibus, internodiis (1,5-)3-6 cm long. Foliae 25-50 mm long. $x$ 10-18 mm lat., pseudofasciculatae, elliptico-lanceolatae, oval-ellipticae, attenuata, pseudopeciolata, apice acuto vel acuminato, discolorae, glabrae vel puberulae. Stipula 2,5-4 mm long., pubescens, laciniis 6-9, 2-6 mm long. Thyrsi in ramis principalibus et lateralibus. Calyx 4partitus, segmentis 1-1,5 mm long., triangular-subulatis, intersegmentis cum appendicibus. Hypantho 1-2 mm, turbinato, glabro. Corolla alba, rosea ad basem, externe micropapillata. Disco integer papilloso. Flos brevistyli: intus corolla, tubo annulari-pilis moniliformis tenuis. Flos longistyli: intus corolla 2 annulari-pilis moniliformis. Capsula 2,5-3,5 mm long. Semina 2,3-2,8 mm long. subcylindrica, dorso convexo, ventre sulcato, strophiolo in linea media. 


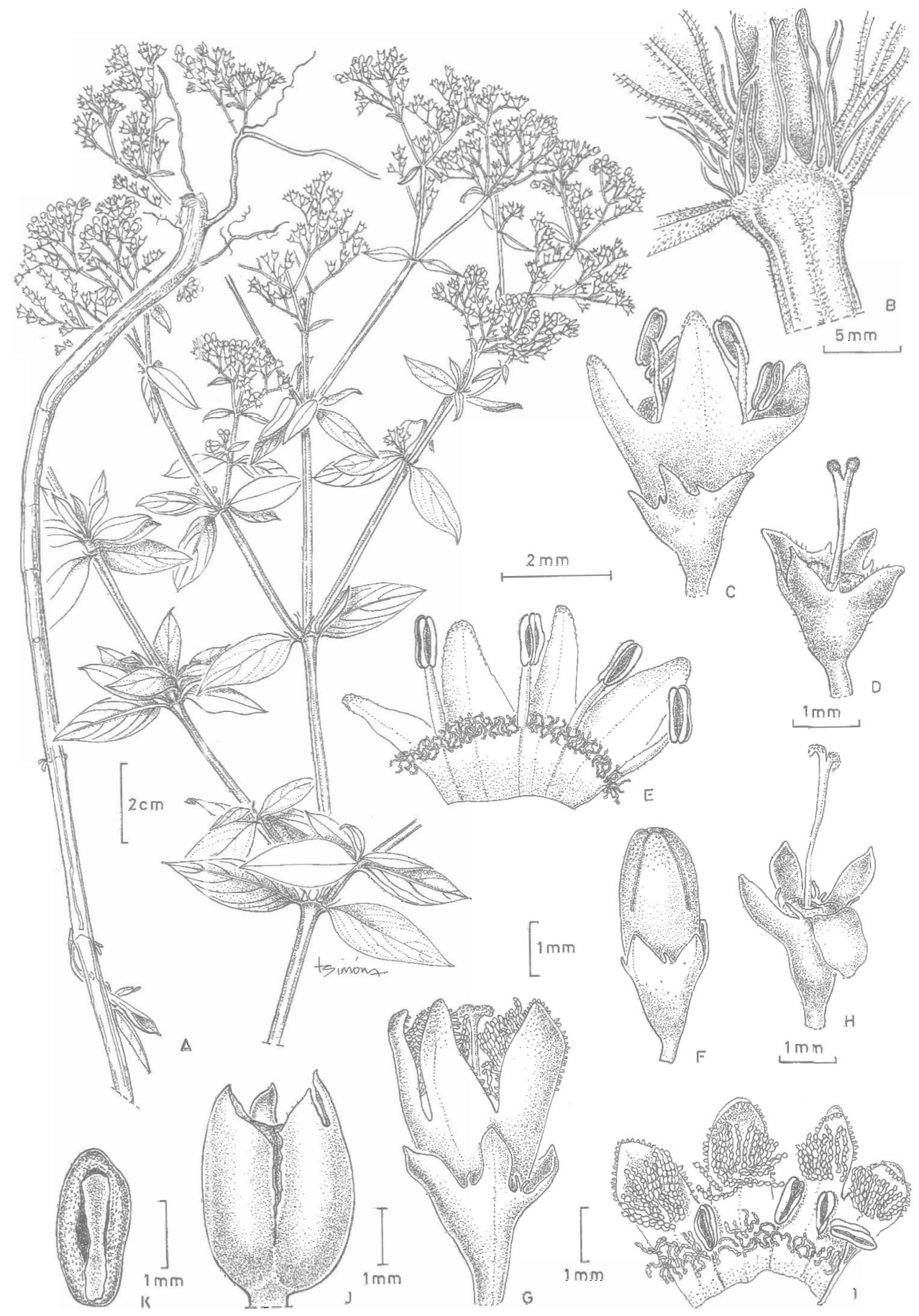

Fig. 1. Galianthe andersonii. A, planta. B, estípula interpeciolar con lacinias. C-E: flor brevistila. C, flor. D, hipanto, cáliz, estilo y estigma. E, corola desplegada. F-I: flor longistila. F, alabastro. G, flor. H, hipanto, cáliz, estilo y estigma. I, corola desplegada. J, fruto. K, semilla. (A-B, F-I, Anderson 8717; C-E, Irwin et al. 23489; J-K, Anderson 8529). 
Holotipo. BRASIL. Minas Gerais. Serra do Espinhaço, $25 \mathrm{~km}$ by road NE of Diamantina, ca. 1.5 km from Rio Jequití, 790-900 m s.m., 12-IV-1973 (fl,fr), W. R. Anderson 8717 (UB). Isotipo: NY!.

Sufrútice muy ramificado, de 60-100 cm alt., tallos tetrágonos, rojizos, de glabros a pubérulos, entrenudos (1,5-) 3-6 cm long., nudos con brotes axilares, pseudoverticilados. Hojas 25-50 mm long. x 10-18 mm lat., elíptico-lanceoladas, de ápice agudo a acuminado, base atenuada en pseudopecíolo, discoloras, más oscuras y brillantes en la haz, glabras a pubérulas, con 5-6 pares de nervios secundarios subopuestos, en relieve en el envés; estípula interpeciolar 2,5-4 mm long., pubescente, con 6-9 lacinias, 2-6 mm long., pubérulas a glabras, rojizas. Inflorescencias tirsoideas terminales. Hipanto 1-2 mm long, turbinado, cáliz 4-partido, segmentos triangular-subulados, glabros, 1-1,5 mm long., dientes intercalares. Corola infundibuliforme, externamente micropapilada, blanca, rosado-pálida hacia la base, de lóbulos más largos que el tubo. Flor brevistila: corola 3-3,5 mm long., superficie interna con un anillo de pelos moniliformes en el tubo, próxima a la base de los lóbulos; anteras 1,2-1,3 mm long., filamentos 1,5 mm long.; estilo $2 \mathrm{~mm}$ long. Flor longistila: corola 2,5-3,3 $\mathrm{mm}$ long., superficie interna con pelos moniliformes, más largos, gruesos y densos en los lóbulos y pelos más cortos y delgados en el tubo, anteras $1 \mathrm{~mm}$ long., filamentos $0,5 \mathrm{~mm}$ long.; estilo $3 \mathrm{~mm}$ long., bífido, ramas estigmáticas lineares. Disco entero, papiloso. Cápsula 2,5-3,5 mm long., subglobosa, de pubérula a glabra. Semilla 2,3-2,8 mm long., subcilíndrica escrobiculada, cara ventral con un surco alrededor del estrofíolo.

Paratipos: BRASIL. Minas Gerais. Serra do Espinhaço, 10 km SW of Diamantina, 3-II-1972 (fl,fr), Anderson et al. 35231 (NY, UB); $18 \mathrm{~km}$ by road SW of Diamantina on road to Curvelo, 10-IV-1973 (fr), Anderson 8529 (NY, UB); 7 km N of São João da Chapada, road to Inhaí, 29-III-1970 (fl,fr), Irwin et al. 28602 (MO, UB); 15 km N of São João da Chapada, 23-III-1970 (fl), Irwin et al. 28120 (UB); $5 \mathrm{~km} \mathrm{~N}$ of Grão Mogol, 18-II-1969 (fl,fr), Irwin et al. 23489 (MO, NY, RB, UB); Parque Estadual do Rio Preto, São Gonçalo do Rio Preto, $18^{\circ} 07^{\prime} 34^{\prime \prime} \mathrm{S} 43^{\circ} 20^{\prime} 28^{\prime \prime} \mathrm{W}, 8$ IV-2000 (fl,fr), Lombardi et al. 3813 (BHCB, CTES).
Distribución geográfica. Se conoce hasta el presente sólo del estado de Minas Gerais. Habita en campos altos entre $700-1400$ m s.m.

Obs. Galianthe andersonii es muy particular por sus tallos rojizos, por sus hojas discoloras con la haz más oscura y brillante, las inflorescencias en todas las ramas, principales y secundarias, y la corola con un ligero tinte rosa. Es afín a Galianthe eupatorioides (Cham. \& Schltdl.) E.L.Cabral, ambas especies están representadas por sufrútices ca. de $1 \mathrm{~m}$ alt., muy ramificados con hojas de forma y tamaño similares, pero G. eupatorioides no presenta tallos rojizos, las estípulas tienen lacinias largas, de 6-12 mm long., y las corolas blancas miden hasta 5,5 mm long. Además es una de las especies, dentro del subgen. Galianthe, con más amplia distribución geográfica (NE de Argentina, Bolivia, Brasil y Paraguay).

Esta especie se dedica al Dr. William R. Anderson que ha contribuido al conocimiento de la tribu Spermacoceae con la monografía del género Crusea Cham. \& Schltdl. y ha coleccionado parte de los ejemplares aquí estudiados.

\section{Galianthe macedoi E.L.Cabral sp. now.}

Fig. 2

Suffrutex 40-80 cm alt., xylopodio; caules simplices, subquadrangulares, fistulosi, glabri. Foliae $55-90 \mathrm{~mm}$ long. x 7-20 mm lat., oblongo-lanceolatae, discolorae, basi truncata vel obtusa, venatio paralelodroma. Semivagina stipularis 6-7 mm long., margine puberula, laciniis 5, 4-12 mm long. Thyrsi late pedunculatis. Hypantho 1,5 mm, turbinato, glabro. Calyx 4-partitus, segmentis 1,5-2 mm long., triangular-subulatis, intersegmentis cum appendicibus. Corolla 4,5-5,5 mm long., alba, externe papillata, lobi dorso apicale dense papilloso. Flos brevistyli: intus corolla, 1 annulari-pilis moniliformis. Flos longistyli: intus corolla 2 annularipilis moniliformis. Disco integer papilloso. Capsula 44,5 mm long., glabra. Semina complanata 2,5-3 mm long.

Holotipo: BRASIL. Goiás. Municipio Jataí, Fazenda Queixada, 10-XII-1948 (fl,fr), A. Macedo 1468 (SP). Isotipos: CTES!, F!, IAC!, NY!, SP!.

Sufrútice 40-80 cm alt., con xilopodio, tallo simple, fistuloso, glabro, subtetrágono. Hojas 55-90 mm long. x 7-20 mm lat., oblongolanceoladas, discoloras, base obtusa o trunca- 


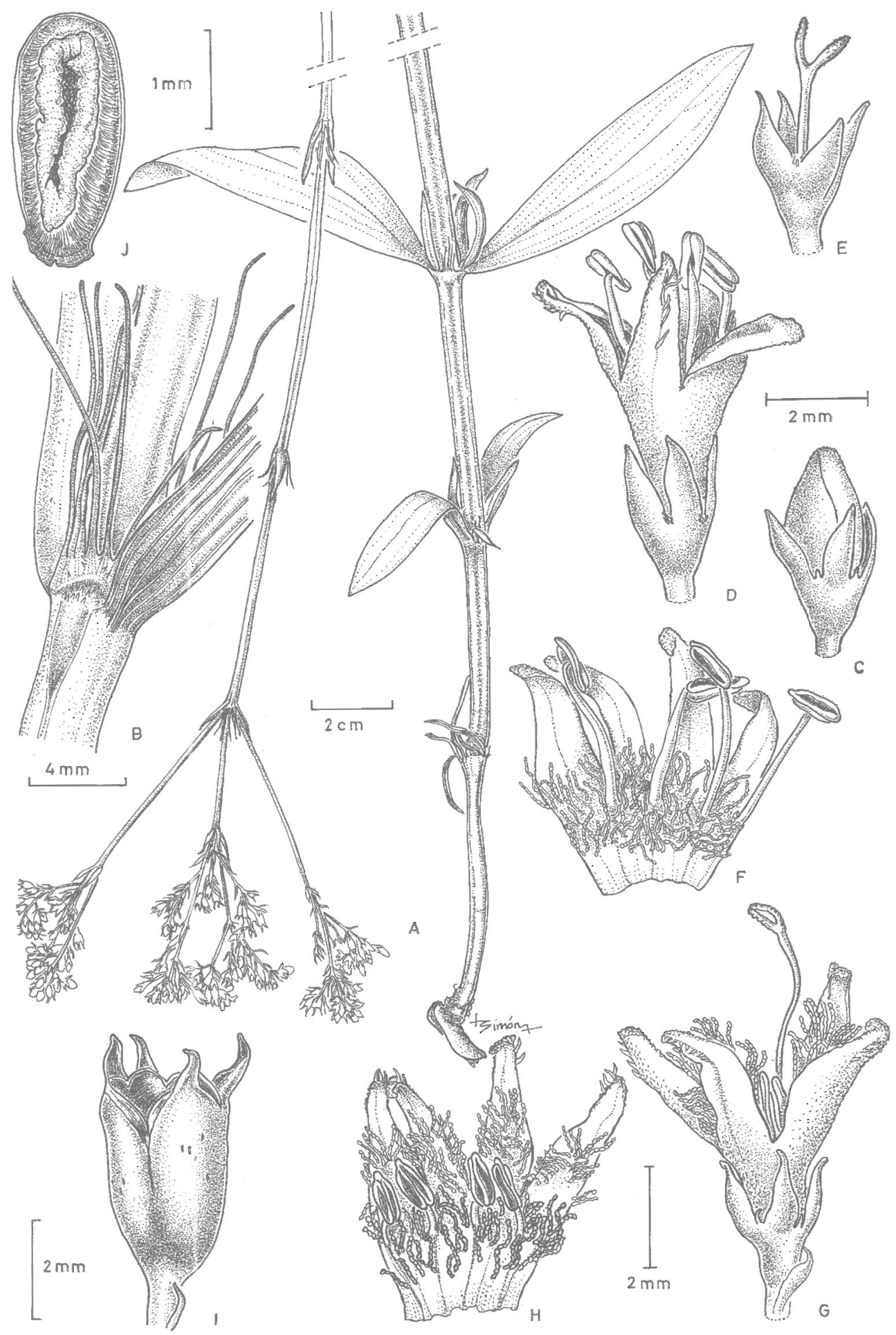

Fig. 2. Galianthe macedoi. A, planta. B, semivaina estipular con lacinias y base foliar señalando parte de venación paralelodroma. C-F: flor brevistila. C, alabastro. D, flor. E, hipanto, cáliz, estilo y estigma. F, corola desplegada. G-H: flor longistila. G, flor. H, corola desplegada. I, fruto. J, semilla.(A-B, F-H, Macedo 1468; C-E, Macedo 1468; I-J, Anderson 9599). 
da y ápice agudo, glabras, venación paralelodroma; estípulas interpeciolares soldadas entre sí, 6-7 mm long., pubérulas, el borde de cada semivaina con 5 lacinias filiformes, la central 8-12 mm long. y las laterales 4-9 mm long. Inflorescencia tirsoide umbeliforme, largamente pedunculada. Hipanto 1,5 mm long., glabro. Cáliz 4-partido, segmentos 1,5-1,8 mm long., triangular-subulados, con dientes intercalares. Corola blanca, 4,5-5,5 mm long., infundibuliforme, lóbulos más largos que el tubo, externamente papilosa, papilas más densas y largas sobre el dorso apical de los lóbulos. Flor brevistila: interior de la corola con un anillo de pelos moniliformes desde la base de los lóbulos hasta la mitad del tubo, el resto papiloso; anteras 1-1,5 mm long., filamentos $3 \mathrm{~mm}$ long.; estilo 3-3,5 mm long., ramas estigmáticas $1 \mathrm{~mm}$. Flor longistila: interior de la corola con pelos moniliformes, más gruesos en la mitad superior del tubo y pelos más delgados en la mitad inferior de los lóbulos; anteras 1-1,3 mm long., filamentos 0,7-1 mm long; estilo 5,5-6,5 mm long., ramas estigmáticas $0,5-0,8 \mathrm{~mm}$ long. Disco entero, papiloso. Fruto 4-4,5 mm long., subcilíndrico, glabro. Semillas 2,5-3 mm long., complanadas dorsiventralmente, con ala estrecha, estrofíolo caduco.

Paratipos: BRASIL. Goiás. ca. 25 km SW of Caiaponia; 800 m s.m., 1-V-1973 (fl,fr), Anderson 9599 (UB, US 2774977, US 2774976).

Distribución geográfica. Todo el material estudiado procede del estado de Goiás, Brasil. Habita en campos bajos cerca de ríos.

Obs. Galianthe macedoi se caracteriza por ser un sufrútice monocaule latifolio y con semillas aladas. La especie más afín es G. grandifolia E.L.Cabral, pero ésta se diferencia de G. macedoi por su porte mayor de 0,80-2,50 m, hojas elípticas a elíptico-lanceoladas de $4-10 \mathrm{~cm}$ long. $\mathrm{x}$ 0,5-4 cm lat., base atenuada en pseudopecíolo, con venación eucamptódroma (Cabral, 1993).

Esta especie está dedicada al Prof. Amaro Macedo, quien ha coleccionado el ejemplar elegido como tipo.

\section{Galianthe peruviana (Pers.) E.L. Cabral comb. nov.}

Spermacoce peruviana Pers. Syn. pl. 1: 124. 1805. "Hab. in Munae ruderatis in Peruvia". Nuevo nombre para Spermacoce corymbosa Ruiz \& Pav. non L.

Spermacoce corymbosa Ruiz \& Pav., nom. illeg., Fl. peruv. 1: 60, tab. 91, fig. a. 1798. "In peruviae montibus ad Muña vicum, in ruderatis et runcationibus Cormillâ et Rinconadâ" Ruiz \& $P a-$ vón holotipo MA, isotipos: B-W!, F!. Non Spermacoce corymbosa L. Sp. pl. ed. 2, 1: 149. 1762.

Borreria ericoides Cham. \& Schltdl. Linnaea 3(4): 326-327. 1828. "In Brasilia aequinoctiali, Sellow", isotipos: LE!, G-DC!.

Borreria corymbosa DC. Prodr. 4: 550. 1830. “In Peruviae montibus, Ceron, Haenke" (G-DC!).

Borreria peruviana (Pers.) L.B.Sm. \& Downs, Sellowia 7: 78-79 y 88. 1956.

Galianthe corymbosa (DC.) E.L.Cabral, Bol. Soc. Argent. Bot. 27(3-4): 241. 1991.

Material estudiado. BOLIVIA. Santa Cruz. Prov. Caballero, $3 \mathrm{~km}$ NE of Abra de Quiñe, 18 $04^{\prime} \mathrm{S}$, 6419'30"W, alt. 2150-2250 m, 31-XII-1995, Nee 46651 (CTES, LPB, NY); Prov. Florida, Samaipata, 1900 m s.m., 29-IX-1981, Beck 7103 A (LPB); 110 km hacia Cochabamba, vegetación alrededor de las ruinas de Samaipata, 1550-1650 m s.m., 23-III-1981, Beck 6765 A (LPB); $5 \mathrm{~km} \mathrm{~W}$ of center of Samaipata, $18^{\circ} 11^{\prime} \mathrm{S}, 6^{\circ} 53^{\prime} \mathrm{W}, 1700-1820 \mathrm{~m}$ s.m., 30-XII-1992, Nee et al. 43413 (CTES, NY, USZ); Samaipata, cerca del cerro de la Patria, 28-II-1994, Jardim et al. 366 (CTES, USZ); Prov. Vallegrande, 5-II-1988, Saldías 196 (USZ). BRASIL. Goiás. Mun. de Alto Paraíso, Nova Roma, 20-II-1991, Pereira et al. 1476 (IBGE); Chapada dos Veadeiros, 16-V-1956, Yale Dawson 14276 (F); ídem, 8-II-1966, Irwin et al. 12311 (MO, RB); ídem, 14-II-1966, Irwin et al. 12825 (RB, UB, US); 21-III-1969, Irwin et al. 24802 (MO, RB); 3-5 km NE of Alto Paraíso, 1100 m s.m., 28-II-1982, Anderson 12479 (MBM); 17 km N of Piranhas on road to Barra do Garças, 23-II-1982, Anderson 12436 (MBM). Minas Gerais. Serra de Moeda, Sellow 1735 (LE); 1844, Weddell s/n (P); ídem, Weddell 1408 (P); Martius 997 (P); 9 km de Itacambira al E de la cidade, na estrada para Montes Claros, afloramiento das rochas 1200 m s.m., 29-XI-1984, Oliveira et al. s/n (SPF 36156); Munic. Jabuticatubas, Lagoa Santa, 14-XII-1971, Semir 561 (UEC); ídem, 15-XII1971, Semir et al. 601 (UEC, SP); ídem, 4-III-1972, Semir et al. 791 (UEC); ídem, 20-VII-1972, Semir et al. 2647 (SP); îdem, 24-VII-1972, Semir et al. 2788 (UEC); ídem, 4-I-1973, Semir et al. 3695 (UEC); ídem, 
29-IV-1973, Semir et al. 4060 (UEC, SP); ídem, 4-III1972, Joly et al. 866 (UEC); ídem, 28-V-1972, Joly et al. 2292 (UEC); Munic. Santana do Riacho, Belo Horizonte, Conceiçao do Mato Dentro, 17-II-1982, Montovani et al. 7792 (UB); ídem, 8-VI-1980, Menezes

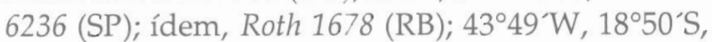
23-IV-1982, Furlan et al. (SPF 32772); Serra do Cipó, 17-I-1972, Hatschbach 28726 (MBM, US); ídem, 19II-1968, Irwin et al. 20535 (NY, UB); ídem, Heringer et al. 21962 (R); ídem, 17-IV-1950, Duarte 2529 (RB); ídem, 14-II-1968, Irwin et al. 19990 (UB, MO); ídem, 19-II-1971, PLK et Urbano 10185 (RB); km 118 da Serra do Cipó, 22-III-1966, Duarte 9622 (CTES, RB); 10-20 km NE de Cardeal Mota, 15-V-1991, Arbo et al. 4153 (CTES, SPF); ídem, 15-V-1990, Arbo et al. 4167 (CTES, SPF); ídem, 8-II-1991, Arbo et al. 4597 (CTES, SPF); 7-12 km N de Santanà do Riacho, camino a Lapinha, 11-II-1991, Arbo et al. 4896 (CTES, SPF); Mun. Urubici, Morro da Igreja, 18-I-1994, Stehmann et al. 1295 (UEC); Pico de Itabirito, 14001600 m s.m., 30-XII-1948, Palacios et al. 3885 (LIL); ídem, 11-II-1968, Irwin et al. 19826 (UB, B); Serra da Matuca, 15-IV-1945, Williams et al. 6640 (LIL); ídem, 22-II-1945, Williams et al. 5568 (K); Munic. Santa Luzia 1100 m s.m., 13-XII-1945, Assis 208 (LIL); Sierra del Espinazo, 1100 m s.m., 10-II-1969, Irwin et al. 22949 (RB, UB, US); ídem, 10-II-1969, Irwin et al. 23010 (UB); entre Campo Alegre e Virgem da Lapa, 2-II-1965, Duarte 8805 (CTES, RB). São Paulo. 1884, Glaziou 17640 (P). PERU. Matthews 1150 (K); Perou-Cochero, Dombey s/n. (P); ídem, 1827, Pavón 36 (P); Quillabamba, 24-IV1957, (F 1627138). Cuzco. Urubamba, Machupicchu, II-1938, Vargas 790 (MO); Urubamba a Machupichu, 2400 m s.m., 22-V-1958, Humbert 30644 (P); Machu Picchu, 2500 m s.m., 19-IV-1954, Hime 784 (F); ídem, 5-V-1982, Peyton et al. 202 A (MO); Huayna Pichu, 2950 m s.m., 1-V-1960, Hoffmann 224 (B); ídem, Vargas 790 (MO); III-1949, Marín 1376 (CTES). Huancavelica. Tayacaja, 28002900 m alt., 14-IV-1954, Tovar 1810 (MO). Huánuco. Camino a Panao, $2650 \mathrm{~m}$ alt., 7-IX-1948, Scolnik 1048 (CORD). Junín. Manto, 11-VII-1961, Woytkowski $6536(\mathrm{MO})$.

Obs. Smith \& Downs (1956: 88) publican Borreria peruviana (Pers.) Sm. \& Downs sin citar material. Al describir esta especie en páginas anteriores, para Santa Catarina (1956: 7879) registran un solo ejemplar, Reitz 2969, el cual lamentablemente no corresponde a esta especie sino a Galianthe reitzii que se describe a continuación en el presente trabajo, pero esto no invalida la nueva combinación propuesta por estos autores.
Posteriormente Cabral (1991) no repara en la ilegitimidad de Spermacoce corymbosa Ruiz \& Pav. y realiza la combinación Galianthe corymbosa (Ruiz \& Pav.) E.L.Cabral.

Chodat \& Hassler (1904) crean Borreria corymbosa DC. f. microphylla Chodat \& Hassl. Al revisar el ejemplar tipo Hassler 4022 proveniente de Tobaty, Paraguay, se comprueba que se trata de Galianthe krausei (Suess.) E.L.Cabral.

Distribución geográfica y hábitat. Vive en campos altos con suelos rocosos en Bolivia (Santa Cruz), Brasil (Goiás, Minas Gerais, São Paulo) y Perú (Cuzco, Huancavelica, Huánuco, Junín). Es la especie del subgen. Galianthe que vive a mayor altura. En Brasil crece en campos cerrados, en suelos con afloramientos rocosos en el planalto central entre $900-1600$ m s.m., en Bolivia es parte de la vegetación puneña en laderas y cumbres de los valles internos andinos del sudoeste de Santa Cruz, entre 1550-2250 m s.m. y en Perú es un componente del estrato herbáceo de las alturas andinas entre 1500-3000 m s.m., generalmente en zonas de pendiente con afloramientos rocosos.

\section{Galianthe reitzii E.L.Cabral sp. nov.}

Fig. 3

Suffrutex ramosissimus 0,20-1 m alt., xylopodio, caules quadrangulares nigrescentes, glabri. Foliae 0,515 (-24) mm long. $x$ 1,5-4 mm lat., sessilae, lineares vel linear-lanceolatae, margine revoluto, utrinque glabrae. Stipula puberula, 1-1,5 mm, irregulari 3 lobulis, collecteri in apice. Thyrsi contracti, pauciflores, in ramis principalibus et lateralibus. Flores dimorphi. Hypantho $1 \mathrm{~mm}$ long:, turbinato, glabro. Calyx 4-partitus, segmentis triangularibus-subulatis, dentibus interlobularis. Flos brevistyli et flos longistyli, corolla 5-6 mm long., alba, externe papillata, intus corolla 2 annularipilis moniliformis, lobulis quam tubum brevioribus. Disco integer papilloso. Fructus, semina non vidi.

Holotipo: BRASIL. Santa Catarina. Municipio Urubici, Serra do Oratorio, Bom Jardim, 9-XII-1958 (fl), R. Reitz \& R. Klein 7688 (HBR). Isotipos: G!, US!.

Arbusto muy ramificado, 0,20-1 m alt., con xilopodio, tallos tetrágonos, glabros, negruzcos, nudos proximales con follaje caduco, entrenudos 2-10 mm. Hojas 0,5-15 (-24) mm long. $x$ 1,5-4 $\mathrm{mm}$ lat., lineares a linear- 


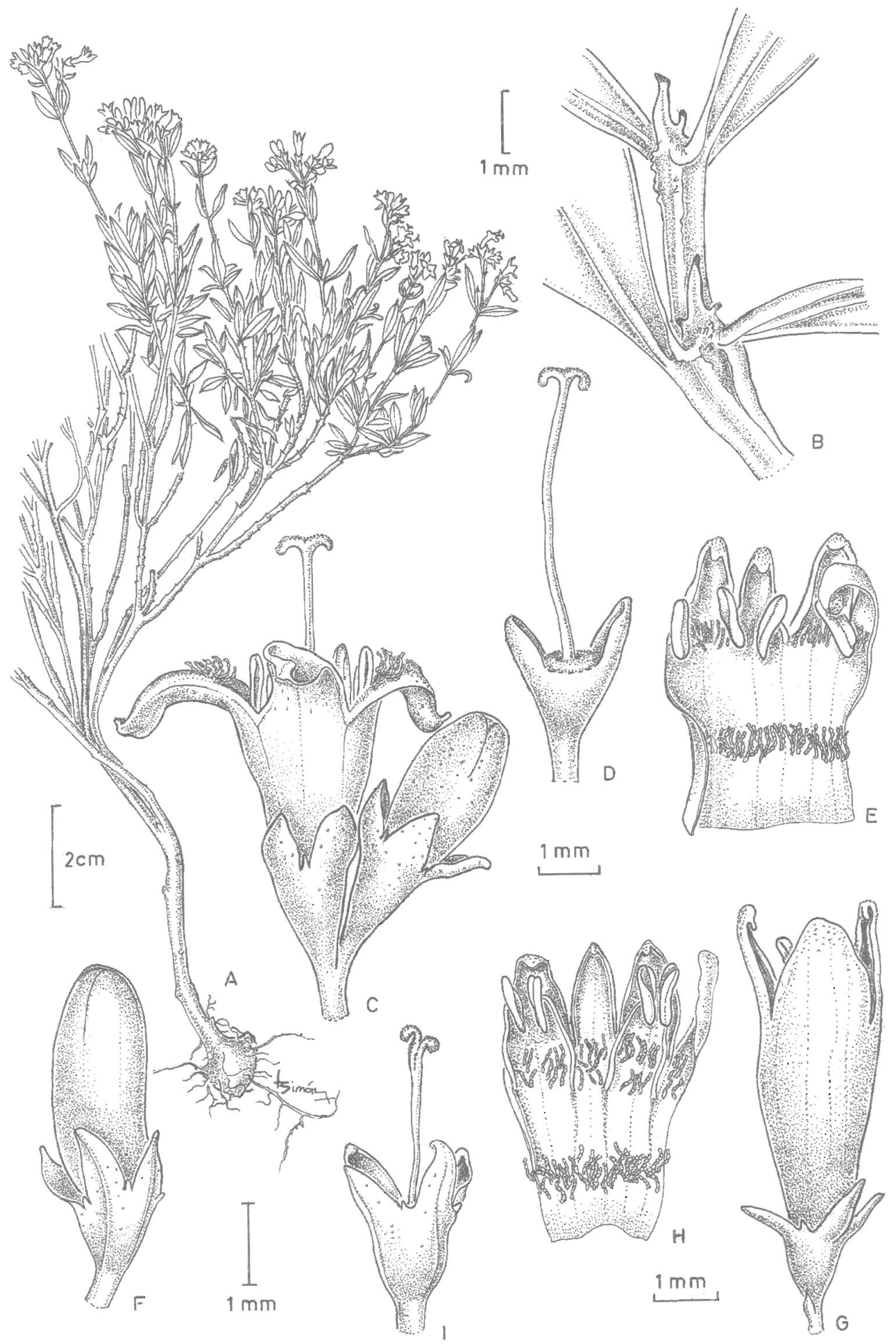

Fig. 3. Galianthe reitzii. A, planta. B, estípula interpeciolar con lacinias. C-E: flor longistila. C, flor y alabastro. D, hipanto, cáliz, estilo y estigma. E, corola desplegada. F-I: flor brevistila. F, alabastro: G, flor: H, corola desplegada: I, hipanto, cáliz, estilo y estigma. (A, B, F-I, Sobral et al. 4830; C-E, Z. Ahumada et al. 3361). 
lanceoladas, sésiles, ápice y base agudos, margen recurvo, coriáceas, nervio principal sobresaliente en el envés, glabras; estípula interpeciolar 1-1,5 $\mathrm{mm}$ long., pubérula, con 3 lacinias subuladas desiguales, 0,5-1,5 mm long. con coléteres apicales. Inflorescencias tirsoideas, paucifloras. Hipanto $1 \mathrm{~mm}$ long., turbinado, glabro. Cáliz 4-partido, segmentos triangulares, 11,5 mm long., dientes intercalares, diminutos. Corola blanca, con lóbulos más cortos que el tubo, por fuera papilosa, en su interior dos anillos de pelos moniliformes, uno con pelos dispersos en la base de los lóbulos y otro con pelos más densos en el tubo, el resto papiloso. Flor brevistila: corola 5-6 mm long., anteras $1 \mathrm{~mm}$, filamentos 1,5 mm; estilo 2,5 mm long., bífido. Flor longistila: corola $6 \mathrm{~mm}$ long., anteras $1 \mathrm{~mm}$, filamentos $1 \mathrm{~mm}$, estilo $6 \mathrm{~mm}$, bífido; disco entero, papiloso. Fruto y semilla no vistos.

Paratipos: BRASIL. Santa Catarina. Municipio Urubici, cuesta a Bom Jardim da Serra, ca. 1000 m s.m., 20-I-1975 (fl), Z. Ahumada et al. 3361 (CTESN, SI); Morro da Igreja, 1822 m s.m., 3-I-1949 (fl), Reitz 2969 (B, HBR, US); Serra do Oratorio, 10 km E de Bom Jardim da Serra, ca. 1200 m s.m., 16-I-1957 (fl), Smith et al. 10151 (CTES, MBM, US); Curral Falso, Bom Jardim, S. Joaquim, 19-II-1959 (fl), Reitz et al. 8419 (HBR, US); Desfiladeiro do Funil, Bom Jardim da Serra, I-1986 (fl), Sobral et al. 4830 (F, MBM); Morro da Igreja, 18-I-1994 (fl), Stehmann et al. 1295 (UEC).

Distribución geográfica. Ha sido coleccionada en Brasil en el SE del estado de Santa Catarina en afloramientos rocosos.

Obs. Galianthe reitzii se caracteriza por ser un sufrútice muy ramificado con hojas pseudoverticiladas, lineares, afin a G. peruviana (Pers.) E.L.Cabral, pero ésta se diferencia por ser de porte más reducido, estípulas interpeciolares diferentes 1,5-2,5 mm long., 35 lacinias 1-3,2 mm long., corolas más pequeñas 2,7-3,2 mm long., con lóbulos \pm de igual longitud que el tubo. En lo que se refiere a la distribución geográfica G. peruviana vive en un área más amplia y G. reitzii aparentemente está restringida al estado de Santa Catarina, Municipio de Urubici, situado en la "Serra Geral", con altitudes que varían entre 1000 a $1822 \mathrm{~m}$ s.m. Esta zona que corresponde al. Parque Nacional de São Joaquim, es considerada la re- gión más fría del Brasil y es el único lugar donde se producen nevadas. La bibliografía consultada señala que la zona se caracteriza por presentar muchas especies endémicas y $G$. reitzii podría ser una de ellas (IBAMA, 1998).

El epíteto específico es un homenaje al P. Raulino Reitz, gran estudioso de la flora catarinense, quien coleccionó el material elegido como tipo.

Borreria G. Mey., es un género pantropical y es el más importante de la tribu Spermacoceae por el número de especies que posee. Autores contemporáneos lo definen como género conflictivo porque se encuentran incluidas entidades de dudosa posición taxonómica, motivo por el cual se inició su actual revisión. Las novedades halladas recientemente son tratadas en el presente trabajo y pertenecen al subgen. Borreria sect. Borreria.

\section{Borreria burchellii E.L.Cabral \& Bacigalupo, stat. et nom. nov.}

Basónimo: Borreria tenella (H.B.K.) Cham. \& Schltdl. var. pumila K.Schum., Fl. bras. 6(6): 5456. 1888. Lectotipo aquí elegido: Brasil, Goiás, [Tocantins], ad Porto Real [Porto Nacional], (fl), Burchell 8679-10 (BR!), non Borreria pumila DC., 1830.

Distribución geográfica. Se conoce sólo el material coleccionado en Tocantins, Brasil.

Material estudiado: BRASIL. Tocantins, Porto Real [Porto Nacional], (fl), Burchell 8679-22 (BR); ídem, Burchell 8657 (BR).

Obs. De los sintipos citados en la descripción original de $B$, tenella var. pumila fueron analizados: Burchell 8679-10 (BR!), Burchell 8679-22 (BR!); Burchell 8657 (BR!). Pohl 2497 no fue localizado. Se seleccionó como lectotipo Burchell 8679-10, por ser un buen ejemplar, completo, con glomérulos con flores abiertas.

2. Borreria crispata (K.Schum.) E.L.Cabral \& Bacigalupo stat. et comb. nov.

Borreria tenella (H.B.K.) Cham. \& Schltdl, var. crispata K.Schum. Fl. bras. 6(6): 54-56. 1888. 
Lectotipo aquí elegido: Brasil, Goiás, May. 1840 (fl,fr), Gardner 4175 (BM!). Isolectotipos: BR!, NY!, P!. Foto F 25582 G-DEL

Distribución geográfica. Especie brasileña propia del cerrado en Bahia, Goiás, Minas Gerais y Tocantins.

Material estudiado: BRASIL. Bahia. Próx. a Cariparé, 113' S 4515'W, 5-IV-1978 (fr), Miranda 252 (RB); próx. a Serra Geral, 4-IV-1978 (fr), Miranda 237 (RB); ídem, 4-IV-1978 (fr), Miranda 232 (RB); $23 \mathrm{~km} \mathrm{~W}$ of Barreiras, 3-III-19728 (fl,fr), Anderson et al. 36557 (UB); Chapadão Ocidental da Bahia, $30 \mathrm{~km}$ N from Correntina, 29-IV-1980 (fr), Harley 21944 (UB, UEC); 46ำ $15^{\prime} \mathrm{W} 14^{\circ} \mathrm{S}, 2-\mathrm{III}-1991$ (fl), Viollati et al. 149 (IBGE); Veredaozinho, Rio Correntina, 133`, 4530` W, 2-II-1967 (fl), Gottsberger 18-2267 (ULM). Goiás. São Domingos, V-1840 (fl,fr), Gardner 4173 (BM). Minas Gerais. Parque Nacional Grande Sertão Veredas, 29-XI-1997 (fl), da Silva et al. 3612 (IBGE, NY); ídem, 28-IV-1999 (fl,fr), Rodriguez da Silva et al. 232 (CTES, IBGE). Tocantins. Chapada das Mangabeiras, 8² $25^{\prime} \mathrm{S} 46^{\circ} 52^{\prime} \mathrm{W}$, (fl,fr), Fonseca 100 (RB).

Obs. Se analizaron los dos sintipos citados en la descripción original de B. tenella var. crispata: Gardner 4175 y Gardner 4173. Se seleccionó como lectotipo Gardner 4175 (BM) por ser el más abundante, completo, con flores y frutos y además cuenta con duplicados, que también fueron analizados.

\section{Borreria tenera DC.}

Candolle, A. P. de, Prodr. 4: 543. 1830. Holotipo: Brasil, 1820, Pohl s/n (G-DC!). Foto F 6711 G-DC.

Borreria pumila Pohl ex DC., Prodr. 4: 543. 1830. Holotipo: Brasil, 1820, Pohl s/n (G-DC!). Foto F 6710 G-DC.

Borreria pumilia Standl., Ann. Missouri Bot. Gard. 27: 342. 1940. Tipo: Panamá, 12-XII-1938 (f1), Allen 822 holotipo: F!, isotipos: MO!, NY!, US! .

Distribución geográfica. Especie recolectada hasta el momento en Panamá, Colombia y Brasil, en campos de suelo arenoso.

Material estudiado: PANAMÁ. San Jose Island, Perlas Archipelago, 6-X-1944 (fl), Johnston 42 (MO). COLOMBIA. Vichada, $10 \mathrm{~km}$ W of Las Gaviotas, 30-XII-1973 (fl), Davidse 5349 (CTES, MO). BRASIL. Distrito Federal, Lago do Paranoa, 2-V-1968 (fl,fr)
Philcox et al. 4855 (K); Chapada de Contagem, northeast, 3-IV-1980 (fl,fr), Plowman 9940 (F). Goiás. inter Torto et Bananal, 9-II I-1895 (fl,fr), Glaziou 21512

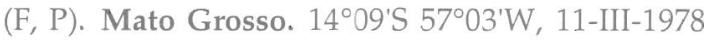
(fl,fr), Becker 031 (RB).

Obs. 1. Cuando de Candolle (1830), describe $B$. tenera la relaciona con $B$. pumila, diferenciándola por ser más glabra, con inflorescencias axilares y con las hojas más cortas. Por el estudio del tipo de B. pumila las autoras reconocen este nombre como sinónimo de $B$. tenera. El análisis del ejemplar tipo de B. pumilio Standl. ha demostrado que esta especie también es un sinónimo de $B$. tenera.

Obs. 2. Los ejemplares citados bajo Borreria tenera por Schumann (1888), corresponden a Borreria paraensis E.L.Cabral \& Bacigalupo (Cabral \& Bacigalupo, 1999), especie caracterizada por ser una hierba glabra con numerosas ramas basales, glomérulos terminales y subterminales y semillas con 13-15 surcos transversales.

\section{Agradecimientos}

Las autoras agradecen a los curadores de los herbarios el préstamo del material estudiado. A C. Zanín la corrección de las diagnosis.

Para la realización de este trabajo se contó con el apoyo económico PMT-PICT 0212, Préstamo BID 802/OC-AR. La primera autora agradece a la Secretaría de Ciencia y Técnica de la UNNE, la financiación de los viajes para estudiar el material de los herbarios extranjeros.

\section{Bibliografía}

BACIGALUPO, N.M. \& E.L.CABRAL. 1996. Infrageneric classification of Borreria (Rubiaceae-Spermacoceae) on the basis of American species. Opera Bot. Belg. 7: 297 308.

CABRAL, E.L. 1991. Rehabilitación del género Galianthe (Rubiaceae). Bol. Soc. Argent. Bot. 27(3-4): 235-249. 1993. Novedades en Galianthe (Rubiaceae). Bonplandia 7(1-4): 1-29.

CABRAL, E.L. \& N.M. BACIGALUPO. 1997. Revisión del género Galianthe subg. Ebelia stat. nov. (Rubiaceae Spermacoceae). Ann. Misso uri Bot. Gärd. 83(4): 857-877. 1999. Estudio de las especies americanas de Borreria serie Laeves (Rubiaceae-Spermacoceae). Darwiniana 37(34): 259.277 .

CANDOLLE, A.P. de. 1830. Prodromus systematis naturalis 
regni vegetabilis (Rubiaceae, Spermacoceae) 4: 538-578.

CHODAT, R. \& E. HASSLER. 1904. Plantae HasslerianaeII. Rubiaceae, sect. Galianthe K. Schum. Bull. Herb. Boissier, sér. 2, 4(2): 341-348.

IBAMA, Instituto Brasileiro do Meio Ambiente, 1998. Unidades de Conservação Federais de Santa Catarina,
Brasil. Parque Nacional de São Joaquim: 4-5.

SCHUMANN, K. 1888. Rubiaceae, Spermacoceae, en Martius, Fl. bras. 6(6): 6-98.

SMITH, L. \& R.J. DOWNS. 1956. Resumo preliminar das Rubiaceas de Santa Catarina. Sellowia 7: 78-79. 1956. Notulae Rubiacearum. Sellowia 7: 87-88. 